\title{
Centralizers of elements in Lie algebras of vector fields with polynomial coefficients
}

\author{
Chapovskyi Y., Efimov D., Petravchuk A.
}

\begin{abstract}
Let $\mathbb{K}$ be an algebraically closed field of characteristic zero, $A=\mathbb{K}\left[x_{1}, \ldots, x_{n}\right]$ the polynomial ring, and $R=\mathbb{K}\left(x_{1}, \ldots, x_{n}\right)$ the field of rational functions in $n$ variables. Denote by $W_{n}=W_{n}(\mathbb{K})$ the Lie algebra of all $\mathbb{K}$-derivations on $A$ (in case $\mathbb{C}$ it is the Lie algebra of all vector fields on $\mathbb{C}^{n}$ with polynomial coefficients). For a given $D \in W_{n}(\mathbb{K})$ the structure of the centralizer $C_{W_{n}(\mathbb{K})}(D)$ depends on the field of constants
\end{abstract}

$$
\text { ker } D=\{\phi \in R \mid D(\phi)=0\}
$$

(here we extend naturally every derivation $D$ of $A$ on the field $R$ ). The case $t r$. $\operatorname{deg}_{\mathbb{K}} \operatorname{ker} D \leqslant 1$ is studied, the structure of the subalgera $C_{W_{n}(\mathbb{K})}(D)$ is characterized, in particular it is proved that if $\operatorname{ker} D$ does not contain any non-constant polynomial, then $C_{W_{n}(\mathbb{K})}(D)$ is finite-dimensional over $\mathbb{K}$. Some results about centralizers of linear derivations in $W_{n}(\mathbb{K})$ are obtained.

\begin{abstract}
Анотація. Нехай $\mathbb{K}$ - алгебраїчно замкнене поле харатеристики нуль, $A=\mathbb{K}\left[x_{1}, \ldots, x_{n}\right]$ - кільце многочленів і $R=\mathbb{K}\left(x_{1}, \ldots, x_{n}\right)$ - поле раціональних функцій від $n$ змінних. Позначимо через $W_{n}=W_{n}(\mathbb{K})$ алгебру Лі всіх $\mathbb{K}$-диференціювань на $A$ (у випадку $\mathbb{C}$ це алгебра Лі всіх векторних полів на $\mathbb{C}^{n}$ з поліноміальними коефіцієнтами). Для заданого $D \in W_{n}(\mathbb{K})$ будова централізатора $C_{W_{n}(\mathbb{K})}(D)$ залежить від поля констант $\operatorname{ker} D=\{\phi \in R \mid D(\phi)=0\}$ (тут ми природнім чином розширюємо кожне диференціювання $D$ на $A$ на поле $R$ ). Досліджено випадок, коли $t r . \operatorname{deg}_{\mathbb{K}} \operatorname{ker} D \leqslant 1$, охарактеризована будова підалгебри $C_{W_{n}(\mathbb{K})}(D)$. Зокрема доведено, що якщо $\operatorname{ker} D$ не містить несталих многочленів, то
\end{abstract}

The first author was supported by Ministry of Education and Science of Ukraine: Grant for perspective development of a scientific direction "Mathematical sciences and natural sciences" at Taras Shevchenko National University of Kyiv, the third author was partially supported by the Ukrainian Government Scientific Research Grant No. 19BF38-02.

Keywords: Lie algebra, derivation, vector field, polynomial ring, centralizer

Ключові слова: Алгебра Лі, диференціювання, векторне поле, кільце многочленів, централізатор

DOI: http://dx.doi.org/10.15673/tmgc.v14i4.2153 
$C_{W_{n}(\mathbb{K})}(D)$ є скінченновимірним над $\mathbb{K}$. Також отримано деякі результати про централізатори лінійних диференціювань в $W_{n}(\mathbb{K})$.

Аннотация. Пусть $\mathbb{K}$ - алгебраически замкнутое поле характеристики нуль, $A=\mathbb{K}\left[x_{1}, \ldots, x_{n}\right]$ кольцо многочленов и $R=\mathbb{K}\left(x_{1}, \ldots, x_{n}\right)$ - поле рациональных функций от $n$ переменных. Обозначим через $W_{n}=W_{n}(\mathbb{K})$ алгебру Ли всех $\mathbb{K}$-дифференцирований на $A$ (в случае $\mathbb{C}$ это алгебра Ли всех векторниых полей на $\mathbb{C}^{n}$ с полиномиальными коэффициентами). Для данного $D \in W_{n}(\mathbb{K})$ строение централизатора $C_{W_{n}(\mathbb{K})}(D)$ зависит от поля констант $\operatorname{ker} D=\{\phi \in R \mid D(\phi)=0\}$ (здесь мы естественным образом продолжаем каждое дифференцирование $D$ на $A$ на поле $R$ ). Изучен случай, когда $t r \cdot \operatorname{deg}_{\mathbb{K}} \operatorname{ker} D \leqslant 1$, охарактеризована подалгебра $C_{W_{n}(\mathbb{K})}(D)$, в частности доказано, что если $\operatorname{ker} D$ не содержит непостоянных многочленов, то $C_{W_{n}(\mathbb{K})}(D)$ бесконечномерен над $\mathbb{K}$. Получены некоторые результаты о централизаторах линейных дифференцирований в $W_{n}(\mathbb{K})$.

\section{INTRODUCTION}

Let $\mathbb{K}$ be an algebraically closed field of characteristic zero (without loss of generality one can assume that $\mathbb{K}=\mathbb{C}$, the field of complex numbers). Denote by $A=\mathbb{K}\left[x_{1}, \ldots, x_{n}\right]$ the polynonial ring and by $R=\mathbb{K}\left(x_{1}, \ldots, x_{n}\right)$ the field of rational functions in $n$ variables.

Recall that a $\mathbb{K}$-linear map $D: A \rightarrow A$ is a $\mathbb{K}$-derivation (or simply a derivation) whenever

$$
D(f g)=D(f) g+f D(g)
$$

for all $f, g \in A$. In case $\mathbb{K}=\mathbb{C}$ every $\mathbb{C}$-derivation can be considered as a vector field on on $\mathbb{C}^{n}$ with polynomial coefficients. We will use this standard correspondence between (polynomial) vector fields and derivations on (polynomial) rings. Any derivation $D$ on $A=\mathbb{K}\left[x_{1}, \ldots, x_{n}\right]$ can be uniquely extended to the derivation $D$ on $R=\mathbb{K}\left(x_{1}, \ldots, x_{n}\right)$ (we use the same notation here) by the rule

$$
D(f / g)=\left(D(f) g-f D(g) / g^{2}\right.
$$

for all $f, g \in A, g \neq 0$.

The Lie algebra $W_{n}(\mathbb{K})$ of all $\mathbb{K}$-derivations on $A$ is of great interest because its finite dimensional subalgebras are closely connected with symmetries of differential equations (recall that any derivation $D$ on $A$ is of the form

$$
D=f_{1}\left(x_{1}, \ldots, x_{n}\right) \frac{\partial}{\partial x_{1}}+\ldots+f_{n}\left(x_{1}, \ldots, x_{n}\right) \frac{\partial}{\partial x_{n}}
$$

for some $f_{i} \in \mathbb{K}\left[x_{1}, \ldots, x_{n}\right]$, where $\frac{\partial}{\partial x_{i}}$ are partial derivatives on $A$ ).

Finite dimensional subalgebras of the Lie algebras $W_{1}(\mathbb{C})$ and $W_{2}(\mathbb{C})$ were classified by S. Lie [4] (more precisely Lie algebras of vector fields with 
analytical coefficients were described in [4]). Analogous problem for $W_{3}(\mathbb{C})$ is open, the problem of classifying all finite-dimensional Lie subalgebras of vector fields from $W_{n}(\mathbb{C}), n \geqslant 4$ is wild [1].

If $D \in W_{n}(\mathbb{K})$, then the centralizer $C_{W_{n}(\mathbb{K})}(D)$ is a subalgebra of $W_{n}(\mathbb{K})$ consisting of all vector fields commuting with $D$. An information about $C_{W_{n}(\mathbb{K})}(D)$ can be useful in many cases. For example, every vector field $D \in W_{n}(\mathbb{C}), D=\sum_{i=1}^{n} f_{i}\left(x_{1}, \ldots, x_{n}\right) \frac{\partial}{\partial x_{i}}$ defines an autonomous system of ODE:

$$
\left\{\begin{array}{c}
\frac{d x_{1}}{d t}=f_{1}\left(x_{1}, \ldots, x_{n}\right) \\
\ldots \\
\frac{d x_{n}}{d t}=f_{n}\left(x_{1}, \ldots, x_{n}\right)
\end{array}\right.
$$

with polynomial coefficients and information about $\operatorname{ker} D$ and $C_{W_{n}(\mathbb{K})}(D)$ can be very useful for searching solutions of (1.1) see, for example [5]. Given $k$ commuting linearly independent over $R$ vector fields on a smooth $n$-manifold $M$, one can construct a local coordinate system on $M$ in which these vector fields are of the form $\frac{\partial}{\partial x_{i}}, i=1, \ldots, k$ (see, e.g. [3, Th. 9.46]). We study centralizers of elements $D \in W_{n}(\mathbb{K})$ in case when ker $D$ (in the field $\left.R=\mathbb{K}\left(x_{1}, \ldots, x_{n}\right)\right)$ is of transcendence degree $\leqslant 1$ over $\mathbb{K}$, i.e. any two rational functions $f, g$ annihilated by $D$ are algebraically dependent over $\mathbb{K}$.

In case $\operatorname{tr}$. $\operatorname{deg}_{\mathbb{K}}$ ker $D=0$ we have $\operatorname{ker} D=\mathbb{K}$ and then $C_{W_{n}}(D)$ is a vector space of dimension $\leqslant n$ over $\mathbb{K}$.

If $t r$. $\operatorname{deg}_{\mathbb{K}}$ ker $D=1$, then by Gordan's theorem (see, e.g. [8]) either ker $D=\mathbb{K}(p)$ or ker $D=\mathbb{K}\left(\frac{p}{q}\right)$, where $p, q$ are irreducible polynomials that are algebraically independent over $\mathbb{K}$.

If ker $D=\mathbb{K}(p)$, then the centralizer $C$ is a module over the ring $\mathbb{K}[p]$ of rank $k, 1 \leqslant k \leqslant n$ and $C$ is either a Lie algebra over $\mathbb{K}[p]$ or it contains an ideal $I$ of rank $k-1$ which is a Lie algebra over $\mathbb{K}[p]$ and $C=I+\mathbb{K}[p] T$ for some derivation $T \in C$ (Theorem 3.1).

In case ker $D=\mathbb{K}(p / q)$ we have that

$$
C=\left(\mathbb{K}(p / q) D+\ldots+\mathbb{K}(p / q) D_{k-1}\right) \cap W_{n}(\mathbb{K})
$$

and $C$ is finite-dimensional over $\mathbb{K}$ (Theorem 3.3).

We use standard notation. Every derivation $D \in W_{n}(\mathbb{K})$ can be uniquely written in the form

$$
D=f_{1}\left(x_{1}, \ldots, x_{n}\right) \frac{\partial}{\partial x_{1}}+\ldots+f_{n}\left(x_{1}, \ldots, x_{n}\right) \frac{\partial}{\partial x_{n}}
$$

for some $f_{i} \in A$. One can show that every nonzero derivation $D$ can be written in the form $D=h D_{0}$, where $D_{0}$ is reduced, i.e. if $D_{0}=h_{1} D_{1}$ for some $D_{1} \in W_{n}(\mathbb{K})$ and $h_{1} \in A$ then $h_{1} \in \mathbb{K}^{*}$. Denote by $\widetilde{W}_{n}(\mathbb{K})$ the Lie 
algebra of all $\mathbb{K}$-derivations of the field $R=\mathbb{K}\left(x_{1}, \ldots, x_{n}\right)$. It is obvious that $\widetilde{W}_{n}(\mathbb{K})$ is a vector space of dimension $n$ over $R$ (with the standard basis $\left.\frac{\partial}{\partial x_{1}}, \ldots, \frac{\partial}{\partial x_{n}}\right)$ but not a Lie algebra over $R$.

A rational function $\varphi \in R=\mathbb{K}\left(x_{1}, \ldots, x_{n}\right)$ is called closed if the subfield $\mathbb{K}(\varphi)$ is algebraically closed in in the field $R$.

\section{Preliminary Results about Centralizers}

Lemma 2.1. Let $D \in W_{n}(\mathbb{K}) \backslash\{0\}, F$ be the field of constants of $D$ in $R$, and $C=C_{\widetilde{W}_{n}(\mathbb{K})}(D)$. Then either

- $C=C_{\widetilde{W}_{n}(\mathbb{K})}(D)=F D$, or

- $C=F D+F D_{2}+\cdots+F D_{k}$ for some $D_{2}, \ldots, D_{k} \in C$ with $D, D_{2}, \ldots, D_{k}$ linearly independent over $R$.

Proof. Note that $C$ is a subalgebra of the Lie algebra $\widetilde{W}_{n}(\mathbb{K})$ over the field $\mathbb{K}$ and $D \in C$. Choose a basis $D, D_{1}, \ldots, D_{k}$ (this includes the case $k=0$ ) for the vector space $R C$ over the field $R$. Every $T \in C$ (note that $C \subseteq R C$ ) can be written in the form $T=r D+r_{2} D_{2}+\ldots+r_{k} D_{k}$ for some $r, r_{i} \in R$. But then the equality $[D, T]=0$ implies $D\left(r_{i}\right)=0, i=1, \ldots, k$, i.e. $r_{i} \in \operatorname{ker} D=F$.

On the contrary, one can note that any element from $F D+\ldots+F D_{k}$ belongs to $C$. Therefore $C=F D+\ldots+F D_{k}$. If $F \subseteq \operatorname{ker} D_{i}$ for all $i \geqslant 2$, then $C$ is not only $k$-dimensional vector space over $F$ but also a Lie algebra over $F$.

Corollary 2.2. Under assumption of Lemma 2.1, if $F=\mathbb{K}$ then $C$ is a $k$-dimensional Lie algebra over the field $\mathbb{K}$.

Example 2.3. Let $D \in W_{n}(\mathbb{K})$ be a linear derivation,

$$
D=f_{1}\left(x_{1}, \ldots, x_{n}\right) \frac{\partial}{\partial x_{1}}+\ldots+f_{n}\left(x_{1}, \ldots, x_{n}\right) \frac{\partial}{\partial x_{n}},
$$

$f_{i}\left(x_{1}, \ldots, x_{n}\right)=D\left(x_{i}\right)=\sum_{j=1}^{n} a_{i j} x_{j}$. Assume also that the Jordan normal form of the matrix $\left(a_{i j}\right)$ is diagonal

$$
\left[\begin{array}{lll}
\lambda_{1} & \ldots & 0 \\
& \ldots & \\
0 & \ldots & \lambda_{n}
\end{array}\right],
$$

where $\lambda_{i}$ are linearly independent over $\mathbb{Z}$ eigenvalues of the matrix $\left(a_{i j}\right)$. Then $C=C_{W_{n}(\mathbb{K})}(D)$ is of rank $n$ over $R$ and has dimension $n$ over $\mathbb{K}$. Indeed, $\operatorname{ker} D=\mathbb{K}$ by [6, Theorem 10.1.2]. Let

$$
L=\left\{\sum_{j=1}^{n} \mu_{j} x_{j} \frac{\partial}{\partial x_{j}} \mid \mu_{j} \in \mathbb{K}\right\} .
$$


One can easily see that $L \subseteq C$ and $\operatorname{rk}_{R} L=n$. Therefore $\operatorname{rk}_{R} C=n$ and $\operatorname{dim}_{\mathbb{K}} C=n$ by Lemma 2.1 .

Lemma 2.4. Let $\mathbb{K}$ be an algebraically closed field of characteristic zero and $L$ an algebraically closed subfield of the field $R=\mathbb{K}\left(x_{1}, \ldots, x_{n}\right)$ with tr.deg $\operatorname{dK}_{\mathbb{K}} L=1$. If $L$ contains a non-constant polynomial from

$$
A=\mathbb{K}\left[x_{1}, \ldots, x_{n}\right] \subset R,
$$

then $L=\mathbb{K}(p)$ for some irreducible polynomial $p \in A$. If $L \cap A=\mathbb{K}$, then $L=\mathbb{K}(p / q)$ for some irreducible polynomials $p, q \in A$ which are algebraically independent over $\mathbb{K}$.

Proof. By Gordan's theorem (e.g. [8, Theorem 3]) we have that $L=\mathbb{K}(\varphi)$ for some rational function $\varphi \in R$.

First let $L \cap A=\mathbb{K}$. The by [7, Corollary 1], we have that $L=\mathbb{K}\left(\frac{p}{q}\right)$ for some irreducible polynomials $p, q$ which are algebraically independent over $\mathbb{K}$. Now let $L \cap A \neq \mathbb{K}$ and $r \in(L \cap A) \backslash \mathbb{K}$. Then $r=F\left(\frac{p}{q}\right)$ or $r=F(p)$ for some rational function $F(t) \in \mathbb{K}(t)$ :

$$
F(t)=\frac{a_{0} x^{m}+a_{1} x^{m-1}+\cdots+a_{m}}{b_{0} x^{n}+b_{1} x^{n-1}+\cdots+b_{n}},
$$

with $a_{i}, b_{j} \in \mathbb{K}, a_{0} b_{0} \neq 0$. If $r=F(p / q)$, then

$$
F(p / q)=\frac{a_{0} p^{m}+\ldots+a_{m} q^{m}}{b_{0} p^{n}+\ldots+b_{n} q^{n}} q^{n-m}
$$

and the numerator and denominator here are homogeneous polynomials in $p$ and $q$ of degree $\max \{m, n\}$. For simplicity assume that $n \geqslant m$ for simplicity. Then

$$
r=F(p / q)=\frac{\left(\alpha_{1} p+\beta_{1} q\right) \ldots\left(\alpha_{n} p+\beta_{n} q\right)}{\left(\gamma_{1} p+\delta_{1} q\right) \ldots\left(\gamma_{n} p+\delta_{n} q\right)}
$$

for some $\alpha_{i}, \beta_{i}, \gamma_{i}, \delta_{i} \in \mathbb{K}$ since the ground field $\mathbb{K}$ is algebraically closed.

Note that the polynomials $\alpha_{i} p+\beta_{i} q$ and $\gamma_{j} p+\delta_{j} q$ are either coprime (when $\left|\begin{array}{ll}\alpha_{i} & \beta_{i} \\ \gamma_{j} & \delta_{j}\end{array}\right| \neq 0$ ) or proportional with a multiplier in $\mathbb{K}^{\star}$ when $\left|\begin{array}{ll}\alpha_{i} & \beta_{i} \\ \gamma_{j} & \delta_{j}\end{array}\right|=0$.

Since the rational function $F(t)$ can be choosen irreducible, the equality (2.1) is impossible because its numerator and denominator are coprime and $r$ is a non-constant polynomial. Thus the case $L=\mathbb{K}(p / q)$ is impossible and $L=\mathbb{K}(p)$ for an irreducible polynomial $p\left(x_{1}, \ldots, x_{n}\right)$.

Proposition 2.5. Let $D_{1} \in \widetilde{W}_{n}(\mathbb{K})$ be such a derivation of the field $R$ that $F=\operatorname{ker} D_{1}$ in $R$ is of transcendence degree 1 over $\mathbb{K}$. Then the centralizer 
$C=C_{\widetilde{W}_{n}(\mathbb{K})}\left(D_{1}\right)$ is a subalgebra of $\widetilde{W}_{n}(\mathbb{K})$ of $\operatorname{rk}_{R} C=k, 1 \leqslant k \leqslant n$, and

$$
C=F D_{1}+F D_{2}+\cdots+F D_{k}
$$

for some $D_{2}, \ldots, D_{k} \in C$. Moreover, either $C$ is a Lie algebra over $F$ of dimension $k$, or $C$ contains an ideal of corank one over $R$ which is a Lie algebra over $F$ of dimension $k-1$.

Proof. By Gordan's theorem (e.g. [8, Theorem 3]) we have that $F=\mathbb{K}(\varphi)$ for some closed rational function $\varphi \in R$. Choose a basis $D_{1}, D_{2}, \ldots, D_{k}$ of $C$ over $R$. As $\left[D_{1}, D_{i}\right]=0, i=1, \ldots, k$, we have $D_{i}\left(\operatorname{ker} D_{1}\right) \subseteq \operatorname{ker} D_{1}$. So $D_{i}(\varphi)=f_{i}(\varphi)$ for some rational functions $f_{i}(t), i=1, \ldots, k$.

If $f_{1}(t)=\cdots=f_{n}(t)=0$, then $F \subseteq \operatorname{ker} D_{i}$ for $i=1, \ldots, k-1$. Therefore, $C=F D_{1}+\ldots+F D_{k}$ is a $k$-dimensional Lie algebra over the field $F$.

Now suppose that $f_{i}(t) \neq 0$ for some $i, 2 \leqslant i \leqslant n$. Then one can easily prove that $f_{i}(\varphi) \neq 0$. Denote by $C_{0}=\{T \in C \mid T(\varphi)=0\}$ the annihilator of the element $\varphi$ in $C$. Since $D_{i}(\operatorname{ker} D) \subseteq \operatorname{ker} D$, we see that $C_{0}$ is an ideal of $C$. We claim that $\operatorname{rk}_{R} C_{0}=k-1$. Indeed, if $T, S \in C \backslash C_{0}$ then $T(\varphi)=g(\varphi)$ and $S(\varphi)=h(\varphi)$ for some nonzero rational functions $g(t)$ and $h(t)$. It now follows that $h(\varphi) T-g(\varphi) S \in C_{0}$ and therefore $\operatorname{rk}_{R} C / C_{0}=1$. Thus we have $\operatorname{rk}_{R} C_{0}=k-1$.

Next, we point out a series $D_{2}, \ldots, D_{n}$ of derivations on the polynomial ring $\mathbb{K}\left[x_{1}, \ldots, x_{n}\right]$ with centralizers $C_{i}=C_{W_{n}}\left(D_{i}\right)$ such that

$$
\operatorname{rank}_{R} C_{i}=n-i+1, \quad i=2, \ldots, n .
$$

We use the known simple derivation from [6, Example 13.4.3].

Lemma 2.6. Let $D_{k}=\frac{\partial}{\partial x_{1}}+\left(1+x_{1} x_{2}\right) \frac{\partial}{\partial x_{2}}+\cdots\left(1+x_{k-1} x_{k}\right) \frac{\partial}{\partial x_{k}}$ be a derivation of the polynomial ring $\mathbb{K}\left[x_{1}, \ldots, x_{n}\right], 2 \leqslant k \leqslant n$. Then

(1) $\operatorname{ker} D_{k}=\mathbb{K}\left[x_{k+1}, \ldots, x_{n}\right]$ for $k<n$ and $\operatorname{ker} D_{n}=\mathbb{K}$;

(2) $C_{k}=C_{W_{n}(\mathbb{K})}\left(D_{k}\right)=\mathbb{K}\left[x_{k+1}, \ldots, x_{n}\right] D_{k}+$

$$
+\mathbb{K}\left[x_{k+1}, \ldots, x_{n}\right] \frac{\partial}{\partial x_{k+1}}+\cdots+\mathbb{K}\left[x_{k+1}, \ldots, x_{n}\right] \frac{\partial}{\partial x_{n}},
$$

for $k<n$ and $C_{n}=\mathbb{K} D_{n}$.

In particular, $\operatorname{rk}_{R}\left(C_{k}\right)=n-k+1$.

Proof. (1) The polynomial ring $A=\mathbb{K}\left[x_{1}, \ldots, x_{n}\right]$ can be considered as the polynomial ring in variables $x_{1}, \ldots, x_{k}$ over the ring $F:=\mathbb{K}\left[x_{k+1}, \ldots, x_{n}\right]$. By [6, Example 13.4.3] $D_{k}$ is a simple derivation of the ring $F\left[x_{1}, \ldots, x_{k}\right]$ (note that $F \subseteq$ ker $D_{k}$ ). Hence the kernel of $D_{k}$ in $F\left[x_{1}, \ldots, x_{k}\right]$ coincides with $F$. Therefore the kernel of the derivation $D_{k}$ in the $\operatorname{ring} A$ coincides with $\mathbb{K}\left[x_{k+1}, \ldots, x_{n}\right]$. 
(2) Let $T \in C=C_{W_{n}(\mathbb{K})}$,

$$
T=f_{1} \frac{\partial}{\partial x_{1}}+\cdots+f_{n} \frac{\partial}{\partial x_{n}} .
$$

Then the equality $\left[T, D_{k}\right]=0$ implies equalities $D_{k}\left(f_{1}\right)=T(1)=0$ and therefore $f_{1} \in \mathbb{K}\left[x_{k+1}, \ldots, x_{n}\right]$,

$$
\begin{array}{lll}
D_{k}\left(f_{2}\right)=x_{1} f_{2}+x_{2} f_{1}, & \ldots, & D_{k}\left(f_{k}\right)=x_{k-1} f_{k}+x_{k} f_{k-1}, \\
D_{k}\left(f_{k+1}\right)=0, & \ldots, & D_{k}\left(f_{n}\right)=0 .
\end{array}
$$

The last $n-k$ equalities imply that

$$
f_{k+1} \in \mathbb{K}\left[x_{k+1}, \ldots, x_{n}\right], \ldots, f_{n} \in \mathbb{K}\left[x_{k+1}, \ldots, x_{n}\right] .
$$

If $f_{1} \neq 0$, then $f_{1} D_{k} \in C_{k}$ and $T-f_{1} D_{k} \in C_{k}$. Therefore without loss of generality one can assume that $f_{1}=0$. But then $D_{k}\left(f_{2}\right)=x_{1} f_{2}$ which is possible only if $f_{2}=0$ because $D_{k}$ is a simple derivation of the ring $F\left[x_{1}, \ldots, x_{k}\right]$. Repeating the arguments one can conclude that

$$
f_{3}=\cdots=f_{k}=0 .
$$

The latter means that

$$
T-f_{1} D_{k} \in \mathbb{K}\left[x_{k+1}, \ldots, x_{n}\right] \frac{\partial}{\partial x_{k+1}}+\cdots+\mathbb{K}\left[x_{k+1}, \ldots, x_{n}\right] \frac{\partial}{\partial x_{n}} .
$$

Taking into account the relation $f_{1} \in \mathbb{K}\left[x_{k+1}, \ldots, x_{n}\right]$ we get the needed statement.

In order to separate factors of a polynomial which belong to the kernel of a derivation we consider the following notions. Let $p \in \mathbb{K}\left[x_{1}, \ldots, x_{n}\right]$ be an irreducible polynomial. A polynomial $f=f\left(x_{1}, \ldots, x_{n}\right)$ will be called $p$-free if $f$ is not divisible by any polynomial in $p$ of positive degree. It can be easily shown that every polynomial $g \in \mathbb{K}\left[x_{1}, \ldots, x_{n}\right]$ can be written in the form $g=g_{0} g_{1}$, where $g_{0}$ is a $p$-free polynomial and $g_{1}=g_{1}(p)$ is a polynomial of $p$ (this includes the case $g_{1}=$ const). The degree in $p$ of the polynomial $g_{1}(p)$ will be called the $p$-degree of $g$ and denoted by $\operatorname{deg}_{p} g$.

Let $p$ and $q$ be algebraically independent irreducible polynomials of the ring $\mathbb{K}\left[x_{1}, \ldots, x_{n}\right]$. A polynomial $f\left(x_{1}, \ldots, x_{n}\right] \in \mathbb{K}\left[x_{1}, \ldots, x_{n}\right)$ will be called $p$ - $q$-free if $f$ is not divisible by any homogeneous polynomial in $p$ and $q$ of positive degree. As earlier one can write every polynomial $g \in \mathbb{K}\left[x_{1}, \ldots, x_{n}\right]$ in the form $g_{0} g_{1}$, where $g_{0}$ is a $p$ - $q$-free polynomial and $g_{1}$ is a homogeneous polynomial in $p, q$. The (total) degree of $g_{1}$ in $p, q$ will be called the $p$ - $q$ degree of $g$ and denoted by $\operatorname{deg}_{p-q} g$.

If $D$ is a derivation on the polynomial ring $\mathbb{K}\left[x_{1}, \ldots, x_{n}\right]$, then $D$ can be written in the form $h D_{0}$, where $D_{0}$ is an irreducible derivation on $\mathbb{K}\left[x_{1}, \ldots, x_{n}\right]$ and $h \in \mathbb{K}\left[x_{1}, \ldots, x_{n}\right]$. We will call $D p$-free if the polynomial $h$ is $p$-free. We summarize all these remarks in the next statement 
Lemma 2.7. Let $D \in W_{n}(\mathbb{K})$ be a nonzero derivation. Then there exist unique (up to a factor from $\mathbb{K}^{\star}$ ) polynomials $f(p, q)$ and $h$ such that

$$
D=f(p, q) h D_{0}
$$

where $D_{0}$ is a reduced derivation, $f(p, q)$ is a homogeneous polynomial in $p, q$ and the polynomial $h$ is $p$-q-free.

\section{Centralizers of elements in $W_{n}(\mathbb{K})$}

Theorem 3.1. Let $D$ be a derivation of the ring $\mathbb{K}\left[x, \ldots, x_{n}\right]$ with the field of constants $F=\operatorname{ker} D$ in $R=\mathbb{K}\left(x_{1}, \ldots, x_{n}\right)$ of the form $F=\mathbb{K}(p)$ for some irreducible polynomial $p$ and let $C=C_{W_{n}(\mathbb{K})}(D)$. Then

(1) If $\operatorname{rk}_{R} C=1$, then $C=\mathbb{K}[p] D_{0}$ for some $p$-free derivation $D_{0}$ with $D=f(p) D_{0}$ for some $f(t) \in \mathbb{K}[t]$;

(2) If $\operatorname{rk}_{R} C \geqslant 2$, then $C$ is either a Lie algebra of rank $k$ over the ring $\mathbb{K}[p]$ or $C$ contains an ideal I of rank $k-1$ that is a Lie algebra over $\mathbb{K}[p]$ and $C=I+\mathbb{K}[p] S$ for some derivation $S \in C$.

Proof. As noted above the derivation $D$ can be written in the form

$$
D=f(p) D_{0},
$$

where $D_{0}$ is a $p$-free derivation and the polynomial $f \in \mathbb{K}[t]$ is uniquely defined by $D$ up to a nonzero multiplier in $\mathbb{K}^{*}$.

(1) First let $\operatorname{rk}_{R} C=1$. Take an arbitrary element $T \in C$. Then $T=\varphi(p) D_{0}$ for some rational function $\varphi \in \mathbb{K}(t)$, where $\varphi(p)=g(p) / h(p)$ for some polyomials $g(t), h(t) \in \mathbb{K}[t]$. Without loss of generality one can assume that $\varphi(t)=g(t) / h(t)$ is a reduced fraction. It follows from the equality $T=\varphi(p) D_{0}$ that $h(p) T=g(p) D_{0}$. Write $D_{0}$ and $T$ in the form

$$
D_{0}=\sum_{i=1}^{n} P_{i}\left(x_{1}, \ldots, x_{n}\right) \frac{\partial}{\partial x_{i}}, \quad T=\sum_{j=1}^{n} Q_{j}\left(x_{1}, \ldots, x_{n}\right) \frac{\partial}{\partial x_{j}},
$$

where $P_{i}, Q_{j} \in \mathbb{K}\left[x_{1}, \ldots, x_{n}\right]$. Suppose that the polynomial $h$ is nonconstant. Since $D_{0}$ is $p$-free, at least one of the coefficients of $D_{0}$ is not a multiple of $h(p)$. Without loss of generality one can assume that $P_{1}$ is such a coefficient. Then it follows from the equality $h(p) T=g(p) D_{0}$ that $h Q_{1}=g P_{1}$. Taking into account the equality $(g(p), h(p))=1$ we see that $h \mid P_{1}$ which gives a contradiction. Therefore $h \in \mathbb{K}^{\star}$ and

$$
\varphi=g\left(x_{1}, \ldots, x_{n}\right) \in \mathbb{K}\left[x_{1}, \ldots, x_{n}\right] .
$$

But then $T=g(p) D_{0}$ and $C=\mathbb{K}[p] D_{0}$ since $T$ was arbitrarily chosen.

(2) Let $\operatorname{rk}_{R} C=k \geqslant 2$. If for each $D_{1} \in C$ we have that $D_{1}(F)=0$, then it is easy to see that $C$ is a Lie algebra of rank $k$ over the ring $\mathbb{K}[p]$. Note that in this case $C$ may not be a free $\mathbb{K}[p]$-module. 
Suppose there exists an element $S \in C$ such that $S(F) \neq 0$. Then $S(p) \neq 0$. Choose $S$ so that the $p$-degree of the polynomial $S(p)$ is minimal.

We claim that for each $T \in C$ the polynomial $T(p)$ is a multiple of $S(p)$. Indeed suppose $S(p)=v(p), T(p)=u(p)$ for some polynomials $v(t), u(t) \in \mathbb{K}[t]$. Write $u(t)=v(t) q(t)+r(t)$ for some polynomials $q(t), r(t)$, where $\operatorname{deg} r(t)<\operatorname{deg} v(t)$. Then $u(p)=v(p) q(p)+r(p)$ and $T-q(p) S \in C$. Since $(T-q(p) S)(p)=r(p)$ and $\operatorname{deg}_{p} r(p)<\operatorname{deg}_{p} S(p)$, we have by the choice of $S$ that $r(p)=0$ and $T-q(p) S$ annihilates the kernel ker $D$. Denote by $C_{0}$ the subalgebra of $C$ of all derivations annihilating $\mathbb{K}[p]$. Then as was shown above $T-q(p) S \in C_{0}$ and $C=C_{0}+\mathbb{K}[p] S$.

Corollary 3.2. If $k=2$ and $C(F) \neq 0$, then $C=\mathbb{K}[p] D_{0}+\mathbb{K}[p] S$ is a free module of rank 2 over $\mathbb{K}[p]$.

Theorem 3.3. Let $D \in W_{n}(\mathbb{K})$ be a derivation with

$$
\text { tr. } \operatorname{deg}_{\mathbb{K}} \operatorname{ker} D=1 \quad \text { and } \quad\left(\operatorname{ker}_{R} D\right) \cap A=\mathbb{K} .
$$

Then

1) $\operatorname{ker} D=\mathbb{K}(p / q)$ for some irreducible algebraically independent polynomials $p, q \in \mathbb{K}\left[x_{1}, \ldots, x_{n}\right]$,

2) the derivation $D$ is of the form $D=h f(p, q) D_{0}$ for some irreducible derivation $D_{0}$ and homogeneous in $p, q$ polynomial $f$ and a $p$-q-free polynomial $h$,

3) the centralizer $C=C_{W_{n}(\mathbb{K})}(D)$ is finite-dimensional over $\mathbb{K}$ being one of the following types:

(a) $C=\mathbb{K}[p, q]_{m} h D_{0}$, where $\mathbb{K}[p, q]_{m}$ is the linear space of homogeneous in $p, q$ polynomials of degree $m=\operatorname{deg}_{p-q} f$, and in particular $\operatorname{dim}_{\mathbb{K}} C=m+1$;

(b) $C=\left(\mathbb{K}(p / q) D+\mathbb{K}(p / q) D_{2}+\cdots+\mathbb{K}(p / q) D_{k}\right) \cap W_{n}(\mathbb{K})$ for some elements $D_{2}, \ldots, D_{k}, k \leqslant n$ in $C$ with $D, D_{2}, \ldots, D_{k}$ linearly independent over the field $R$.

Proof. By Lemma 2.4 we have that $\operatorname{ker} D=\mathbb{K}(p / q)$ for some irreducible algebraically independent over $\mathbb{K}$ polynomials $p, q$. By Lemma 2.7 there exist unique (up to a nonzero factor from $\mathbb{K}$ ) polynomials $f(p, q)$ and $h$ such that $D=f(p, q) h D_{0}$, where $D_{0}$ is a reduced derivation, $f(p, q)$ is a homogeneous polynomial at $p, q$ and the polynomial $h$ is $p$ - $q$-free.

First, let $\operatorname{rk}_{R} C=1$. Then for any $D_{1} \in C$ we have that $D_{1}=r D_{0}$ for some $r \in A$ (because $D_{0}$ is a reduced derivation). As mentioned above, $r=f_{1} h_{1}$ for some homogeneous polynomial $f_{1}(p, q)$ in $p, q$ and a $p$ - $q$-free 
polynomial $h_{1}$. By the choice of $D_{1}$ we have that

$$
0=\left[D, D_{1}\right]=\left[f_{1} h_{1} D_{0}, f h D_{0}\right] .
$$

The last relation implies the equality

$$
D_{0}\left(f h /\left(f_{1} h_{1}\right)\right)=0 .
$$

By Lemma $2.4 f h /\left(f_{1} h_{1}\right)=u(p, q) / v(p, q)$ for some homogeneous polynomials $u, v$ in $p, q$ with $\operatorname{deg} u=\operatorname{deg} v$. Hence

$$
h f v=h_{1} f_{1} u,
$$

where $f v$ and $f_{1} u$ are homogeneous in $p, q$ and $h, h_{1}$ are $p$ - $q$-free polynomials. Recall that the factorization of a polynomial as a product of a homogeneous in $p, q$ and a $p$ - $q$-free polynomial is unique up to a factor from $\mathbb{K}^{\star}$. Hence $h_{1}=h c, c \in \mathbb{K}^{*}$, and $f v=c^{-1} f_{1} u$. Then

$$
\operatorname{deg}_{p-q} f=\operatorname{deg}_{p-q} f_{1}=m .
$$

This implies the relation

$$
D=f_{1} h_{1} D_{0} \in \mathbb{K}[p, q]_{m} h D_{0} .
$$

Since $D_{1}$ was arbitrarily chosen in $C$, we have the inclusion

$$
C \subseteq \mathbb{K}[p, q]_{m} h D_{0} .
$$

It is easy to see that $\mathbb{K}[p, q]_{m} h D_{0} \subseteq C$ and therefore $C=\mathbb{K}[p, q]_{m} h D_{0}$.

Further, let $\operatorname{rk}_{R} C=k \geqslant 2$. Choose a basis $D, D_{2}, \ldots, D_{k}$ of $C$ over $R$. Then by Proposition 2.5

$$
C=\left(\mathbb{K}(p / q) D+\mathbb{K}(p / q) D_{2}+\ldots+\mathbb{K}(p / q) D_{k}\right) \cap W_{n}(\mathbb{K}) .
$$

We will show by induction on $k$ that the centralizer $C=C_{W_{n}(\mathbb{K})}(D)$ is finite-dimensional over $\mathbb{K}$.

For $k=1$ (i.e. in case $\operatorname{rk}_{R} C=1$ ) this was proved above, so we may assume that $k \geqslant 2$.

Denote for convenience $D_{1}=D$. Then every element $D_{i}$ can be written in the form $D_{i}=\sum_{j=1}^{n} P_{i j} \frac{\partial}{\partial x_{j}}$ for some polynomials $P_{i j} \in A, i=1, \ldots, k$. Take an arbitrary element $T$ of the centralizer $C$ and write down it in the form $T=\sum_{i=1}^{k} \alpha_{i} D_{i}$ for some rational functions $\alpha_{i} \in R$. On the other hand, the same derivation can be written in the standard form $T=\sum_{j=1}^{n} Q_{i} \frac{\partial}{\partial x_{i}}$ for some polynomials $Q_{1}, \ldots, Q_{n} \in A$. Consider the derivations $D_{1}, \ldots, D_{k-1}, T$ and denote by $\left(P_{i j}^{\prime}\right)$ the polynomial matrix whose first $k-1$ rows consist of coefficients of derivations $D_{1}, \ldots, D_{k-1}$ and the $k$-th row is of the form $\left(Q_{1}, \ldots, Q_{n}\right)$, i.e. $P_{i j}^{\prime}=P_{i j}$ and $P_{k j}^{\prime}=Q_{j}$ for $i=1, \ldots, k-1, j=1, \ldots, n$. 
Consider the minor $\delta=\delta_{i_{1}, \ldots, i_{k}}$ on arbitrarily chosen columns $i_{1}, \ldots, i_{k}$ of the matrix $\left(P_{i j}\right)$ and the analogous minor $\mu=\mu_{i_{1}, \ldots, i_{k}}$ on the same columns of the matrix $\left(P_{i j}^{\prime}\right)$. Since $T=\sum_{i=1}^{k} \alpha_{i} D_{i}$, we have obviously the equality $\mu=\alpha_{k} \delta$.

Repeating the arguments from the proof of Lemma 2.4 one can show that there exist homogeneous polynomials $u, v$ in $p, q$ with $\operatorname{deg}_{p-q} u=\operatorname{deg}_{p-q} v$ such that $\alpha_{k}=u / v$. It follows from the equality $\mu=\alpha_{k} \delta$ (written in the form $v \mu=u \delta$ ) that $\operatorname{deg}_{p-q} \mu=\operatorname{deg}_{p-q} \delta$. Moreover, these polynomials have the same $p$ - $q$-free part up to a factor from $\mathbb{K}^{\star}$ because of the equality $v \mu=u \delta$ mentioned above. We can assume that $p-q$-free parts of $u$ and $v$ are identical, denote their common value by $h$. Let $M_{1}, \ldots, M_{s}$ be all the $(k \times k)$-minors of the matrix $\left(P_{i j}^{\prime}\right)$ enumerated in an arbitrary way, so $s=\left(\begin{array}{l}n \\ k\end{array}\right)$. Then they are polynomials from $A$. Let $m=m_{i}$ be the $p-q$ degree of the minor $M_{i}$ and $f_{i}$ the corresponding homogeneous polynomial which is a $p-q$-part of the minor $M_{i}$. We assign to the derivation $T$ the sequence of homogeneous polynomials $\theta(T)=\left(f_{1}, \ldots, f_{s}\right)$ of degrees $m_{1}, \ldots, m_{s}$ correspondingly. Consider the map

$$
\theta: C \rightarrow N=\mathbb{K}[p, q]_{m_{1}} \times \ldots \times \mathbb{K}[p, q]_{m_{s}},
$$

where $m_{i}$ are $p$-q-degree of the minor $M_{i}, i=1, \ldots, s$. The mapping $\theta$ is $\mathbb{K}$-linear and acts from $C$ to $N$, note that $\operatorname{dim}_{\mathbb{K}} N<\infty$. Obviously ker $\theta$ consists of such derivations $T$ for which all the minors of order $k$ are zeroes. But then

$$
T \in\left(\mathbb{K}(p / q) D_{1}+\ldots+\mathbb{K}(p / q) D_{k-1}\right) \cap W_{n}(\mathbb{K})=C_{k-1} .
$$

Therefore, $\operatorname{dim} C / C_{k-1}<\infty$. By inductive assumption the subspace $C_{k-1}$ is finite dimensional over the field $\mathbb{K}$. Therefore $\operatorname{dim}_{\mathbb{K}} C<\infty$.

\section{Centralizers of some linear Derivations}

A derivation $D=\sum_{i=1}^{n} P_{i} \frac{\partial}{\partial x_{i}}$ will be called linear if all the polynomial $P_{i}$ are linear forms in $n$ variables, i.e. $P_{i}=\sum_{j=1}^{n} a_{i j} x_{j}, a_{i j} \in \mathbb{K}$. The linear derivation $D=\sum_{i, j=1}^{n} a_{i j} x_{j} \frac{\partial}{\partial x_{j}}$ is determined by the square matrix $\left(a_{i j}\right)$ of order $n$ and if $D^{\prime}=\sum_{i, j=1}^{n} b_{i j} x_{j} \frac{\partial}{\partial x_{j}}$, then $\left[D, D^{\prime}\right]$ is linear and defined by the matrix $\left(c_{i j}\right)=\left[\left(a_{i j}\right),\left(b_{i j}\right)\right]$. Therefore all the linear derivation form a subalgebra of $W_{n}(\mathbb{K})$ isomorphic to the general linear algebra $g l_{n}(\mathbb{K})$, which (for simplicity) will also be denoted by $g l_{n}(\mathbb{K})$ 
Let $D=\sum_{i=1}^{n} a_{i j} x_{j} \frac{\partial}{\partial x_{j}} \in W_{n}(\mathbb{K})$ be a linear derivation. Then one can consider two centralizers:

$$
C_{0}=C_{g l_{n}(\mathbb{K})}(D) \quad \text { and } \quad C=C_{W_{n}(\mathbb{K})}(D),
$$

Evidently, $C_{0} \subseteq C$. The structure of the centralizer $C_{0}$ is well-known because it consists of all linear derivations defined by the matrices commuting with the matrix $\left(a_{i j}\right)$. How to find the centralizer of a given matrix $\left(a_{i j}\right)$ is a classical problem of linear algebra. It was solved many years ago (see, e.g. [2, Chapter VIII, §2]). Therefore it is interesting to study the case when $C=C_{0}$ because we will then have a complete description of the centralizer $C=C_{W_{n}(\mathbb{K})}(D)$.

In Theorem 4.2 we will present a necessary condition and a sufficient condition for a linear derivation $D$ to satisfy the equality

$$
C_{W_{n}(\mathbb{K})}(D)=C_{g l_{n}(\mathbb{K})}(D)
$$

(unfortunately those conditions do not coincide).

Lemma 4.1. Let $D=\sum_{i=1}^{n} f_{i} \frac{\partial}{\partial x_{i}}$ and $T=\sum_{i=1}^{n} g_{i} \frac{\partial}{\partial x_{i}}$ be two elements of $W_{n}(\mathbb{K})$, where $f_{i}=f_{i}\left(x_{1}, \ldots, x_{n}\right), g_{i}=g_{i}\left(x_{1}, \ldots, x_{n}\right) \in \mathbb{K}\left[x_{1}, \ldots, x_{n}\right]$. Then the derivations $D$ and $T$ commute if and only if $D\left(g_{i}\right)=T\left(f_{i}\right)$ for all $i=1, \ldots, n$.

Proof. It is obvious that $D T=T D$ if and only if $D T\left(x_{i}\right)=T D\left(x_{i}\right)$ for all $i=1, \ldots, n$. But $T\left(x_{i}\right)=g_{i}\left(x_{1}, \ldots, x_{n}\right)$ and $D\left(x_{i}\right)=f_{i}\left(x_{1}, \ldots, x_{n}\right)$ for all $i=1, \ldots, n$. Hence $D\left(g_{i}\right)=T\left(f_{i}\right)$.

Theorem 4.2. Let $D=\sum_{i, j=1}^{n} a_{i j} x_{j} \frac{\partial}{\partial x_{i}}$ be a linear derivation of the polynomial ring $K\left[x_{1}, \ldots, x_{n}\right]$, and $\lambda_{1}, \ldots, \lambda_{n}$ the eigenvalues of the matrix $\left(a_{i j}\right)$. Then the following statements hold:

(1) If the eigenvalues $\lambda_{1}, \ldots, \lambda_{n}$ are linearly independent over $\mathbb{Z}$, then $C_{W_{n}(K)}(D)=C_{g l_{n}(K)}(D)$.

(2) If $C_{W_{n}(K)}(D)=C_{g l_{n}(K)}(D)$, then the eigenvalues $\lambda_{1}, \ldots, \lambda_{n}$ are linearly independent over $\mathbb{N} \cup\{0\}$.

Proof. (1) Suppose that the eigenvalues $\lambda_{1}, \ldots, \lambda_{n}$ of the matrix $\left(a_{i j}\right)$ are linearly independent over $\mathbb{Z}$. Take any $T \in C_{W_{n}(\mathbb{K})}(D)$,

$$
T=\sum_{i=1}^{n} f_{i} \frac{\partial}{\partial x_{i}},
$$


where $f_{i} \in A=\mathbb{K}\left[x_{1}, \ldots, x_{n}\right]$. Without loss of generality one may assume that the matrix $\left(a_{i j}\right)$ is diagonal of the form

$$
\left(a_{i j}\right)=\left(\begin{array}{cccc}
\lambda_{1} & 0 & \cdots & 0 \\
0 & \lambda_{2} & \cdots & 0 \\
\cdots & \cdots & \cdots & \cdots \\
0 & 0 & \cdots & \lambda_{n}
\end{array}\right)
$$

(the eigenvalues $\lambda_{1}, \ldots, \lambda_{n}$ are pairwise distinct, so the matrix $\left(a_{i j}\right)$ is diagonalizable). In view of this assumption the derivation $D$ is of the form

$$
D=\sum_{i=1}^{n} \lambda_{i} x_{i} \frac{\partial}{\partial x_{i}}
$$

Evidently, $D\left(f_{i}\right)=T\left(\lambda x_{i}\right)=\lambda_{i} f_{i}$, i.e. the coefficients $f_{i}$ of the derivation $T$ are Darboux polynomials for $D$ with cofactors $\lambda_{i}, i=1, \ldots, n$. Moreover, $D\left(x_{i}\right)=\lambda_{i} x_{i}, i=1, \ldots, n$. But then

$$
D\left(f_{i} / x_{i}\right)=\frac{D\left(f_{i}\right) x_{i}-f_{i} D\left(x_{i}\right)}{x_{i}^{2}}=0, \quad i=1, \ldots, n
$$

i.e. the rational function $f_{i} / x_{i}$ belongs to the kernel of $D, i=1, \ldots, n$. Since all the eigenvalues $\lambda_{1}, \ldots, \lambda_{n}$ are linearly independent over $\mathbb{Z}$ it follows from [6, Theorem 10.1.2] that $f_{i} / x_{i}=\mu_{i} \in \mathbb{K}, i=1, \ldots, n$. The latter means that

$$
T=\sum_{i=1}^{n} \mu_{i} x_{i} \frac{\partial}{\partial x_{i}} \in g l_{n}(\mathbb{K})
$$

and therefore $C_{W_{n}(K)}(D)=C_{g l_{n}(K)}(D)$.

(2) Suppose that

$$
C_{W_{n}(K)}(D)=C_{g l_{n}(K)}(D) .
$$

This implies that ker $D=\mathbb{K}$. Indeed, if $h \in \operatorname{ker} D \backslash \mathbb{K}$, then $h D \in C_{W_{n}(\mathbb{K})}(D)$ and the derivation $h D$ is obviously nonlinear. Hence by [6, Theorem 10.1.1] the eigenvalues $\lambda_{1}, \ldots, \lambda_{n}$ are linearly independent over $\mathbb{N}_{0}$.

Remark 4.3. Note that the derivation

$$
D=x_{1} \frac{\partial}{\partial x_{1}}+2 x_{2} \frac{\partial}{\partial x_{2}}
$$

on the polynomial ring $K\left[x_{1}, x_{2}\right]$ with eigenvalues 1,2 has nonlinear elements in its centralizer in $W_{2}(\mathbb{K})$, for example $x_{1}^{2} \frac{\partial}{\partial x_{2}}$. So the condition $(2)$ is not sufficient. 


\section{REFERENCES}

[1] V. M. Bondarenko, A. P. Petravchuk. Wildness of the problem of classifying nilpotent Lie algebras of vector fields in four variables. Linear Algebra Appl., 568:165-172, 2019, doi: 10.1016/j.laa.2018.07.031.

[2] F. R. Gantmacher. The theory of matrices. Vol. 1. AMS Chelsea Publishing, Providence, RI, 1998.

[3] J. M. Lee. Introduction to smooth manifolds, volume 218 of Graduate Texts in Mathematics. Springer, New York, second edition, 2013.

[4] S. Lie, F. Engel. Theorie der Transformationsgruppen. 3. Number 3. Teubner (Leipzig), 1893.

[5] J. Nagloo, A. Ovchinnikov, P. Thompson. Commuting planar polynomial vector fields for conservative Newton systems. Communications in Contemporary Mathematics, 22(04):1950025, 2020.

[6] A. Nowicki. Polynomial derivations and their rings of constants. Uniwersytet Mikołaja Kopernika, Toruń, 1994.

[7] A. P. Petravchuk, O. G. Iena. On closed rational functions in several variables. Algebra Discrete Math., (2):115-124, 2007.

[8] A. Schinzel. Polynomials with special regard to reducibility, volume 77 of Encyclopedia of Mathematics and its Applications. Cambridge University Press, Cambridge, 2000, doi: 10.1017/CBO9780511542916.

Received: November 11, 2021, accepted: November 29, 2021.

Chapovskyi Y.

Department of Algebra and Computer Mathematics, Faculty of Mechanics and Mathematics, Kyiv Taras Shevchenko University, 64, Volodymyrska street, 01033 Kyiv, UKRAINE

Email: safemacc@gmail.com

Efimov D.

Department of Algebra and Computer Mathematics, Faculty of Mechanics and Mathematics, Kyiv Taras Shevchenko University, 64, Volodymyrska street, 01033 Kyiv, UKRAine

Email: d_efimov@knu.ua

Petravchuk A.

Department of Algebra and Computer Mathematics, Faculty of Mechanics and Mathematics, Kyiv Taras Shevchenko University, 64, Volodymyrska street, 01033 KYIV, UKRAINE

Email: apetrav@gmail.com

ORCID: 0000-0003-0371-7771 